\title{
LOS MAESTROS MAYORES DE FORTIFICACIÓN Y EL ACCESO AL CUERPO DE FRANCISCO CANSINO Y PATIÑO: UN PROYECTO DE HOSPITAL MILITAR PARA LA CIUDAD DE SEVILLA
}

\author{
THE MASTER BUILDERS OF FORTIFICATIONS CORPS \\ AND THE ACCESS OF FRANCISCO CANSINO Y PATIÑO: \\ A MILITARY HOSPITAL PROJECT FOR THE CITY OF \\ SEVILLE
}

\author{
IGNACIO J. LÓPEZ HERNÁNDEZ \\ Universidad de Almería. España \\ ORCID: 0000-0001-6917-4664 \\ ignajlh@ual.es
}

Las dificultades operativas del Real Cuerpo de Ingenieros Militares a la hora de coordinar a pie de obra sus numerosas obras motivaron desde su fundación la institución de figuras subalternas entre las que destacaron los maestros mayores de fortificación. Con la reorganización del cuerpo en el siglo XIX, sus funciones, cualificación y estatus quedaron definidos de forma general, llegando a integrar la plantilla de empleados del Cuerpo en calidad de civiles. En este trabajo se analiza el proceso de definición corporativa del maestro mayor de fortificación a lo largo de sus diferentes ordenanzas y a partir del análisis del caso particular de Francisco Cansino y Patiño, del que se documenta su proyecto para un hospital militar como examen de acceso a la plaza de Sevilla.

Palabras clave: arquitectura; fortificación; hospitales; ingeniería militar; maestros mayores.

The struggles of the Spanish Corps of Military Engineers to coordinate its numerous works motivated the creation of different subordinate employees such as the master builders of fortifications -maestros mayores de fortificación-. With the reorganisation of the Corps in the $19^{\text {th }}$ century, the functions, qualifications and status of this figure were defined, being included as part of the Corps as civilians. This paper analyses how the master builders of fortifications were integrated into the structure of the Corps of Military Engineers by analysing the different ordinances and by studying the case of the master builder of Seville Francisco Cansino y Patiño and his admission test, a military hospital project.

Keywords: architecture; fortification; hospitals; military engineer; master builders. 


\section{LA DEFINICIÓN DE LA FIGURA DEL MAESTRO MAYOR DE FORTIFICACIÓN}

En 1711 tenía lugar la fundación del Real Cuerpo de Ingenieros Militares bajo el auspicio del general Jorge Próspero de Verboom con el fin de institucionalizar la figura del ingeniero militar y con ello dotar a la corona de una herramienta eficiente para el control defensivo de sus extensos dominios ${ }^{1}$. Sus integrantes, antes diseminados por otros cuerpos del ejército, constituyeron desde entonces un arma sujeta a rangos, formación y organización autónoma. Sin embargo, pese a los numerosos procesos de reforma y reorganización al que se le sometió durante todo el siglo XVIII, nunca dejó de ser un cuerpo facultativo con oficiales especializados en la proyección de las obras, pero sin personal subalterno integrado en él que contribuyera a la buena marcha de estas. En relación a ello la Real Ordenanza e Instrucción de 4 de julio de 1718 para los Ingenieros, y otras personas ya disponía que para la ejecución de las obras de fortificación se habría de acudir puntualmente a diferentes profesionales en coordinación con los oficiales de la Real Hacienda:

"Por lo que mira a los Sobreestantes, Maestros, Oficiales, y Peones, reglará el Intendente de acuerdo con el Ingeniero, y con la intervención del Contador, los que serán menester para la obra; y el jornal diario con que a cada uno se le ha de acudir [...] bien entendido, que los Sobreestantes que hubieren de atender a la buena construcción de la obra, además de los Ingenieros Subalternos que han de vigilar al mismo fin, han de ser nombrados por el Ingeniero Director, y a su entera satisfación; y así se ha de nombrar por el Intendente el Sobreestante, o Ministro que de su parte hubiere de intervenir"'.

Con la introducción de instrucciones y ordenanzas complementarias, se creó la figura del maestro mayor de Reales Obras de Fortificación entre algunas comandancias peninsulares y ultramarinas, si bien en ningún momento constituyó un cuerpo subalterno ${ }^{3}$. Tampoco estos profesionales llegaron a ser suficientes para atender la demanda, sobre todo en los amplios territorios de ultramar donde

${ }^{1}$ El trabajo de referencia para conocer la constitución y ordenación del cuerpo durante todo el siglo XVIII es CAPEL, Horacio; SÁNCHEZ, Joan-Eugeni y MONCADA, Omar: De Palas a Minerva. La formación científica y la estructura institucional de los ingenieros militares en el siglo XVIII. Barcelona, 1988. Sobre Verboom puede consultarse la monografía de MUÑOZ CORBALÁN, Juan Miguel: Jorge Próspero Verboom: ingeniero militar flamenco de la monarquía hispánica. Madrid, 2015.

${ }^{2}$ Instrucciones y ordenanzas de 4 de julio de 1718. Madrid, 1720, art. 36.

${ }^{3}$ RAMOS PÉREZ, Demetrio: "Los maestros mayores de reales obras de fortificación y los sobrestantes: Conocimientos exigidos, funciones, sueldos y aspiraciones sociales", en Memoria del III Congreso Venezolano de Historia. T. II. Caracas, 1979, pp. 525-552. 
la poca disponibilidad de efectivos obligó a una continua improvisación ${ }^{4}$. Así, fue recurrente que la construcción de las obras, proyectadas previamente por los oficiales ingenieros, se sometiera al otorgamiento de una contrata, por lo general bajo una subasta a la cual concurrían maestros de obras particulares con sus propias cuadrillas. Estos debían ajustarse a un pliego de condiciones establecido por los oficiales, quedando en todo momento bajo la supervisión y control de los militares. Sin embargo, este sistema siempre adoleció de continuos problemas de operatividad, traduciéndose en numerosos pleitos entre la administración y los contratistas, debidos a retrasos en las obras, incumplimiento de condiciones o mala ejecución de los trabajos, casi siempre consecuencia de supeditarse tales responsabilidades a personal no suficientemente cualificado ${ }^{5}$.

Esta situación fue poco a poco revirtiéndose con las nuevas disposiciones que enmarcaron la estructura y organización del cuerpo durante el siglo XIX. La primera de ellas tuvo lugar en 1803 mediante la promulgación de la Ordenanza que Su Majestad manda observar en el servicio del Real Cuerpo de Ingenieros de $1803^{6}$. Esta normativa tuvo un impacto decisivo en la configuración del cuerpo durante todo el ochocientos, esbozando las atribuciones y organización que se detallarían en regulaciones subsiguientes. Quizá, el aspecto más notable de la nueva ordenanza radicaba en la reconstitución de un cuerpo con funciones exclusivamente militares, que tendería a hacerse cada vez más autónomo en operatividad ${ }^{7}$. Prueba de ello es el mandato establecido en el artículo 25 del

${ }^{4}$ A pesar de constituir la mayor parte de la extensión geográfica de la Corona española, la cuota de ingenieros militares en el continente americano siempre fue mucho menor que la peninsular. GUTIÉRREZ, Ramón y ESTERAS, Cristina: Arquitectura y Fortificación. De la ilustración a la independencia americana. Madrid, 1993, pp. 69-99.

${ }_{5}$ Especialmente problemático fue el contexto americano, donde la escasez de facultativos, recursos y maestros cualificados condicionaron la buena marcha de los proyectos de fortificación y obras militares. Un caso especialmente elocuente tuvo lugar durante los más de cuarenta años que duró la construcción del castillo de San Severino de Matanzas. Véase CASTILLO MELÉNDEZ, Francisco: La defensa de la Isla de Cuba en la segunda mitad del siglo XVII. Sevilla, 1986, pp. 328-357; y HERNÁNDEZ GODOY, Silvia: El Castillo de San Severino: insomne caballero del puerto de Matanzas (1689-1898). Matanzas, 2006. Este problema también lo he tratado en un periodo previo en LÓPEZ HERNÁNDEZ, Ignacio J.: "Proyectos y proceso para la fundación y defensa de la ciudad cubana de San Carlos de Matanzas. Fortificación y urbanismo entre 1681 y 1693”, Anuario de Estudios Atlánticos, 64, 064-002, 2018, pp. 1-15.

${ }^{6}$ Ordenanza que S. M. manda observar en el servicio del Real Cuerpo de Ingenieros. Madrid, 1803.

7 Una de las consecuencias directas de esta ordenanza fue la eliminación de las funciones en el campo de las obras públicas que hasta entonces tuvieron los ingenieros militares. CANTERA MONTENEGRO, Jesús: "Aportaciones singulares de los ingenieros militares a la obra civil”, Revista de Historia Militar, número extraordinario, 2012, pp. 13-32; y CAPEL, Horacio: "Los ingenieros militares: su formación científica y su intervención en las 
Reglamento Tercero en el que se cita que "en todas las Plazas de consideracion habrá un Maestro mayor, que ademas de las circunstancias de integridad y desinteres, tenga un perfecto conocimiento de toda especie de obras y materiales, pues ha de ser el principal Zelador, y responsable de todo lo irregular y contrario a la mejor consistencia y metodo que se note por el Ingeniero en la construccion y sistema que haya establecido al presenciar la traza".

Del mismo modo se designaba el sistema de acceso a la plaza mediante la evaluación directa del director subinspector de ingenieros de los pretendientes a través de un examen del que se debían acreditar conocimientos en los principios de las "Matemáticas, levantamiento de Planos, nivelaciones, corte de perfiles, principales partes de la Arquitectura, práctica de obras y conocimientos de los materiales del pais"9. Una vez aprobada la elección por parte del ingeniero general, obtendría un nombramiento oficial desde la corte, con sueldo fijado en función de lo que en el momento se determinase. Cabe aclarar que estos sujetos procedieron de la sociedad civil y que, a pesar de integrarse en la organización del cuerpo, no llegaron a alcanzar por esta vía empleo militar ${ }^{10}$. A pesar de ello, estos maestros mayores tuvieron reglado su propio uniforme y se les concedió un considerable estatus al equiparárseles en la normativa a arquitectos de las reales academias de San Fernando y San Carlos ${ }^{11}$. De la misma forma y con condiciones parecidas se creó la plaza de sobrestante mayor al que se le otorgó responsabilidades contables para lo que se les requería "buena letra, y saber los principios necesarios de Aritmética"12. Ambas plazas junto con la de escribiente determinaron los primeros empleos subalternos del arma de

obras públicas", en Antiguas obras hidráulicas. Actas del Seminario México 1988. Madrid, 1991, pp. 507-541. Un caso particular a partir de entonces lo constituyó Cuba durante los dos primeros tercios del siglo XIX, donde los ingenieros militares, contraviniendo esta y otras normativas, se integrarán en las administraciones civiles que coordinaron la construcción de las obras públicas en la isla. Véase LÓPEZ HERNÁNDEZ, Ignacio J.: "El Cuerpo de Ingenieros Militares y la Real Junta de Fomento de la Isla de Cuba. Obras públicas entre 1832 y 1854", Espacio, Tiempo y Forma, Serie VII, Historia del Arte, 4, 2016, pp. 483-508.

8 Ordenanza que S. M. manda observar en el servicio..., op. cit., art. 25, Reglamento Tercero, pp. 129-130.

9 Ibidem, art. 26, p. 130.

${ }^{10}$ MURO MORALES, José Ignacio: "Ingenieros militares en España en el siglo XIX. Del arte de la guerra en general a la profesión del ingeniero en particular", Scripta Nova, Revista Electrónica de Geografía y Ciencias Sociales, vol. VI, 119 (93), 2002. Edición digital sin paginar: http://www.ub.edu/geocrit/sn/sn119-93.htm (Consultado el 19/10/2018).

${ }^{11}$ El maestro mayor debía vestir "casaca y calzon azul, buelta y chupa encarnada, con galon estrecho de plata al canto de esta, cuello anteado y boton blanco". Ordenanza que $S$. M. manda observar en el servicio..., op. cit., art. 27, p. 131.

12 Ibidem, art. 28, p. 131. 
ingeniero que se vieron acotados en sus funciones en las siguientes disposiciones ${ }^{13}$. Fue el caso del Reglamento para el servicio del Cuerpo de Ingenieros del Ejército de 1839, en el que aparece de nuevo la figura del maestro mayor como delegado a pie de obra de los intereses de la administración del ejército ${ }^{14}$. Ahora se le atribuyeron tareas concretas como reconocimientos del terreno durante el proceso de proyección de las obras ${ }^{15}$, supervisión de las adquisiciones de materiales ${ }^{16}, y$ control como individuo de mayor rango de los empleados de las obras que se acometieran por cuenta de la administración ${ }^{17}$.

Sin embargo, para la ajustada aplicación de este último reglamento se requirió de la aparición de nuevas figuras no contempladas por las anteriores disposiciones en las filas del cuerpo. Se dictaba así por Real Decreto de 26 de mayo de 1840 el Reglamento [...] para la organizacion de los empleados subalternos del arma de ingenieros con el que se establecieron dos clases de empleados subalternos ${ }^{18}$. Compusieron los empleados de primera clase los maestros mayores de fortificación y los maestros de obras, mientras que fueron de segunda clase los celadores de fortificación y los conserjes. Los primeros se ocuparon de auxiliar la dirección de las obras militares y los de segunda clase de vigilar su ejecución y conservación

${ }^{13}$ La creación de estas figuras obedecía como vemos a la necesidad de un mayor control en la ejecución de las obras, motivo por el que se le dedicaría en la misma ordenanza un título a "los asientos y contratos para la construccion de obras". En él se debatiría de nuevo sobre la conveniencia de llevar a cabo las obras por contrata o a costa de operarios empleados por la Real Hacienda. Mientras que la primera "es la mas opuesta a su buena construccion y permanencia, por lo dificil que es impedir los fraudes y abusos", el método de ejecución a través de la administración "sin embargo de ser el mas a propósito para lograr la mejor solidez y consistencia, tiene el incoveniente de ser muchas veces el mas costoso por el crecido número de empleados que exige para zelar y atender el gran número de ramos que se abrazan por este método". Se concluye por tanto que la combinación de ambos sistemas resultaría el más beneficioso, subdividiendo las fases "dando destajos o asientos parciales para todo lo que sea acopio de materiales, labra [...] y quanto no pertenezca al ramo de construccion o fabrica, que deberá hacerse siempre por administración”. Sin embargo, no deja de contemplarse en las ordenanzas la posibilidad de que todo en su conjunto se ejecutara mediante asiento, para lo que también delimita actuaciones muy específicas. Ordenanza que S. M. manda observar en el servicio..., op. cit., art. 1, título II, pp. 133-134.

${ }_{14}$ Reglamento para el servicio del Cuerpo de Ingenieros del Ejército en lo tocante a los proyectos, direccion y contabilidad de las obras de fortificacion y edificios militares que tiene a su cargo aprobados por Su Majestad en 5 de junio de 1839. La Habana, 1856.

15 Ibidem, cap. 3, art. 14, p. 7.

${ }^{16}$ Ibid., cap. 8, pp. 18-20.

17 Ibid., cap. 9, pp. 20-25.

18 Reglamento aprobado por Su Majestad por Real Decreto de 26 de mayo de 1840 para la organizacion de los empleados subalternos del arma de ingenieros. Madrid, 1840. 
y llevar la contabilidad de los trabajos ${ }^{19}$. Según el reglamento, se entenderían como empleos permanentes nombrados por Real Orden solo los de los maestros mayores y los celadores, mientras que los maestros de obras y conserjes dependían de la contratación directa del ingeniero general. Ya con carácter temporal, y siempre que las obras se ejecutaran por cuenta de la administración, podrían integrar las filas del cuerpo subalterno empleados en clase de aparejadores, alarifes y maestros de carpintería y herrería, entre otros, quienes quedarían subordinados a los anteriores ${ }^{20}$.

En cuanto a la organización específica de los maestros mayores de fortificación, se establecieron diecisiete plazas en toda la península, las cuales a su vez se dividieron en cuatro de primera clase, radicadas en las ciudades de mayor valor estratégico, con sueldo de 9.000 reales anuales, y trece de segunda, con salario de $7.000^{21}$. El acceso a ambas plazas seguiría quedando supeditado al examen de los candidatos en los que se acreditaran sobradamente los requisitos y conocimientos indicados en 1803, si bien ahora este procedimiento se sometió a su vez a una doble evaluación. De todos los candidatos que concurrieran al examen, el subinspector o comandante de la plaza debía elevar al ingeniero general una relación detallada con los tres mejores candidatos con la valoración personal de estos mismos jefes, así como las notas de los examinadores. El mejor valorado sería designado, previa aprobación del gobierno, por el ingeniero general. En la elección, se tendrían en cuenta acreditaciones académicas, sobre todo en el caso de que el aspirante tuviera título de arquitecto por la Academia de San Fernando ${ }^{22}$. Aun no siendo así, se abundaba en lo dispuesto en la ordenanza de 1803 sobre seguir considerando a estos maestros mayores de fortificación merecedores de "las mismas preeminencias y regalías que los Arquitectos de las Academias de San Fernando y San Cárlos"23. A su vez, la promoción de segunda a primera clase, quedaba inicialmente sujeta a la vacante de algunas de las cuatro dispuestas, en cuyo caso sería accesible para el maestro mayor de segunda clase de mayor antigüedad, siempre que estuviera dispuesto a mudarse a la cabecera de su plaza.

La distribución de estas últimas quedó desde esta disposición fijada también territorialmente. Las cuatro maestrías mayores de primera clase fueron establecidas en las ciudades de Madrid, Barcelona, Cádiz y Coruña; por su parte las de segunda en Sevilla, Zaragoza, Valladolid, Figueras, Badajoz, Granada, Melilla,

\footnotetext{
${ }^{19}$ Ibidem, art. 1, p. 5.

${ }^{20}$ Ibid., art. 3, p. 6.

${ }^{21}$ Ibid., art. 5, p. 6.

${ }^{22}$ Ibid., arts. 6-9, pp. 6-7.

${ }^{23}$ Ibid., art. 11, p. 8.
} 
Peñón, Pamplona, Cartagena, Peñíscola y Mallorca ${ }^{24}$. Pese a ello, los maestros mayores, quedaron sujetos a una constante movilidad por toda la extensión de su demarcación, fijando estas ciudades la sede de su residencia. Pese a la rápida aplicación de la ordenanza se tardarían años en ocupar todas las plazas. Así, en 1841, al año siguiente de su promulgación, solo se habían cubierto diez de las diecisiete plazas $^{25}$, mientras que una década después se llegaban a los quince ${ }^{26}$. Solo hasta 1853 no se ocuparon todas las plazas previstas, aunque por entonces varias de las de maestro mayor de segunda clase se habían trasladado a otras ciudades como Santa Cruz de Tenerife o Valencia, en detrimento de otras como Granada o Peñíscola ${ }^{27}$.

\section{FRANCISCO CANSINO Y PATIÑO, MAESTRO MAYOR DE FORTIFICACIÓN DE SEVILLA}

La figura del maestro de obras de fortificación, a pesar de su poca visibilidad, constituyó una herramienta fundamental en la compleja y eficiente administración que el Cuerpo de Ingenieros desarrolló a partir de la segunda mitad del siglo XIX. De esto dan cuenta los casos particulares de cada uno de ellos, de los que en buena parte se conservan sus hojas de servicio como integrantes subalternos del cuerpo. A través de esta documentación es posible advertir su formación teórica en el momento de su acceso al cuerpo por medio de sus pruebas y exámenes de capacitación. Fue el caso del maestro mayor de fortificación de Sevilla Francisco Cansino y Patiño, quien sustituyó al fallecido Juan Manuel Caballero en 1857. Cansino y Patiño nació en Sevilla el primero de julio de $1827^{28}$. Su formación tuvo lugar en la Escuela de Nobles Artes de Sevilla, donde superó los dos cursos de las enseñanzas de Maestros de Obras entre 1851 y 1853 en los que su disposición y resultados académicos le valieron la distinción de mejor alumno ${ }^{29}$.

${ }^{24}$ Estado del número, clase, distribucion por distritos militares y puntos de habitual residencia de los empleados subalternos del Cuerpo Nacional de Ingenieros. Ibid., anexo 1.

25 "Empleados Subalternos del Arma en las Direcciones, Subinspecciones y Comandancias exentas", en Cuerpo de Ingenieros. Estado General. Año de 1841. Madrid, 1841, pp. 43-48.

26 "Empleados para ausiliar la direccion de las obras", en Estado del Cuerpo de Ingenieros del Ejército en primero de enero de 1851. Madrid, 1851, pp. 104-105.

27 "Empleados para auxiliar la direccion de las obras", en Estado del Cuerpo de Ingenieros del Ejército en $1^{\circ}$ de enero de 1853. Madrid, 1853, pp. 98-98.

28 AGMS (Archivo General Militar de Segovia), sección 1, leg. 992, Empleados Subalternos del Arma. Francisco de Paula Cansino y Patiño.

${ }^{29}$ DE BESA GUTIÉRREZ, Rafael: La Academia y su museo: Criterios académicos en la Gestión de los Fondos del Museo de Bellas Artes de Sevilla. Tesis Doctoral, Sevilla, 2016, pp. 370-371. 
Ya egresado, ocupó interinamente la plaza de maestro mayor de Fortificación de Sevilla desde el 20 de enero de 1857 durante la enfermedad de Caballero. No obstante, el acceso oficial no tuvo lugar hasta la superación de la prueba de pensado, en la que los aspirantes debían elaborar el proyecto de un edificio militar, que sería evaluado por el comandante y el ingeniero general. En este caso, se ha localizado el ejercicio entregado por Cansino el 27 de julio de 1857 , formado por una memoria escrita ${ }^{30}$ y tres planos ${ }^{31}$ con el proyecto de un hospital militar para 344 enfermos para la ciudad de Sevilla. A la convocatoria de la misma plaza concurrió asimismo el maestro de obras Manuel Portillo, quien dos días después firmó una memoria para una academia militar para la misma ciudad ${ }^{32}$. El ejercicio de Cansino fue el mejor valorado, por lo que el ingeniero general lo propuso para la plaza en agosto de aquel mismo año, lo que fue aprobado por Real Orden el 23 de septiembre con el sueldo de 7.000 reales anuales $^{33}$. La siguiente noticia de la que se tiene constancia corresponde al 20 de julio de 1859 cuando obtuvo real licencia para casarse con Salvadora $\mathrm{Mu}$ rieta y Valera Valcárcel ${ }^{34}$.

Tras siete años de servicio continuado en el cuerpo, el propio maestro mayor solicitó el retiro voluntario aduciendo problemas de salud, lo que se hizo efectivo el 31 de diciembre de $1864^{35}$. Una vez recuperado, en mayo de 1876 tuvo la voluntad de volver al puesto con motivo del deceso del maestro mayor de fortificación de la ciudad Manuel Requejo Acosta ${ }^{36}$. La solicitud contó con algunas recomendaciones e informes favorables dirigidos al ingeniero general José de Orozco y Zúñiga. No obstante, para cuando Orozco recibió la solicitud ya había sido cesado del cargo, de modo que la instancia pasó al negociado tercero del cuerpo que emitió informe desfavorable por imposibilitarlo la Ley de Retiros vigente ${ }^{37}$.

Al margen de estas noticias, poco más se ha podido conocer de este personaje. Hasta la fecha tan solo ha sido reconocido como autor de la sala de San

30 AGMS, sección 1, leg. 992, Memoria del Hospital Militar para esta Plaza con un número de 344 enfermos, Francisco Cansino y Patiño, 27-7-1857, ff. 11r-21r.

31 AGMM (Archivo General Militar de Madrid), Cartoteca, SE-9/22, SE-9/23 y SE9/24, Proyecto de un Hospital Militar para 344 camas destinado al servicio de esta plaza, Francisco Cansino y Patiño, 27-7-1857.

32 AGMS, sección 1, leg. 992, Memoria y Presupuesto, Manuel Portillo, 29-7-1857.

${ }_{33}$ Ibidem, Servicios Militares y Méritos que ha contraido, f. $2 \mathrm{r}$.

${ }^{34}$ Ibid., Real licencia de matrimonio a favor de Francisco Cansino y Patiño, 20-71859 , f. 3 r.

${ }_{35}$ Ibid., Empleados Subalternos del Arma. Francisco de Paula Cansino y Patiño, f. 1r.

${ }^{36}$ Ibid., Francisco Cansino y Patiño al ingeniero general, 17-5-1876, f. 8r-v.

${ }^{37}$ Ibid., Informe del Negociado $3^{\circ}, 22-5-1876$. 
José del hospital de la Caridad ${ }^{38}$, figurando como ayudante en otras obras de la ciudad $^{39}$. Resulta complicado adscribirle alguna obra durante el periodo en que ocupó la plaza de maestro mayor de fortificaciones, pues debió quedar supeditado a los proyectos de los oficiales del arma. Esta situación debió cambiar a partir de 1865 cuando cesó en el cargo. Así aparece anunciado desde el año siguiente como maestro mayor de obras y caminos particulares -e incluso como arquitecto- en algunas ediciones de la Guía de Sevilla de Manuel Gómez Zarzuela ${ }^{40}$. Como contribución al conocimiento de este maestro mayor, se da aquí a conocer la formación artística y capacitación técnica demostradas por el proyecto de hospital militar con el que obtuvo el acceso a la plaza.

\section{PROYECTO DE UN HOSPITAL MILITAR PARA LA CIUDAD DE SEVILLA}

El proyecto de hospital de Cansino no constituyó nunca una propuesta ni necesidad real de la comandancia sevillana, sino que, como era habitual en otras academias y cuerpos, solo debía ser un ejercicio teórico y práctico con el que evaluar los conocimientos del candidato. A pesar de ello, el maestro mayor no obvia en él el supuesto de construirlo en la ciudad, a fin de ajustarlo a las condiciones topográficas y presupuestarias de la zona. Por último, en relación con su ubicación, Cansino aludía a que debía levantarse extramuros de la ciudad, a fin de beneficiarse tanto de la higiene y calidad del aire circundante, como del abaratamiento del solar.

Según el maestro mayor, el carácter militar del edificio obligaba a la adaptación de la tipología hospitalaria convencional a las demandas de orden, disciplina y jerarquía del ejército, pues "el soldado enfermo [...] se debe considerar como si fuera un correccional, pues la esperiencia nos acredita que son mas parecidas a las de esos hombres que no las de un enfermo" 41 . Esta premisa condicionaría la distribución y accesos de un complejo edilicio compuesto en planta por un gran rectángulo de 440 por 240 pies $(122,5$ x 66,8 m). La figura la compondrían cuatro crujías de una sola altura ocupadas en su mayoría por salas para camas de enfermos y convalecientes. El ingreso al hospital se haría por un acceso principal al centro de uno de los lados mayores, en torno a cuyo

${ }^{38}$ VALDIVIESO GONZÁLEZ, Enrique y SERRERA, Juan Miguel: El Hospital de la Caridad de Sevilla. Sevilla, 1980, pp. 30-31.

39 VILLAR MOVELLÁN, Alberto: Arquitectura del regionalismo en Sevilla, 19001935. Sevilla, 1979, p. 149.

${ }^{40}$ GÓMEZ ZARZUELA, Manuel: Guía de Sevilla: su provincia, Arzobispado, Capitanía General, Tercio Naval, Audiencia Territorial y Distrito Universitario. Sevilla, 1866, p. XXXVIII.

${ }^{41}$ AGMS, sección 1, leg. 992, Memoria del Hospital Militar..., doc. cit., f. 13r. 
vestíbulo se repartirían salas para el cuerpo de guardia, sala de reconocimientos, cuarto del oficial y conserje e $^{42}$.

El gran patio generado por esta estructura acogería dos edificios a doble altura de planta cuadrada de 130 pies de lado. Ambos quedarían conectados por una galería porticada doble de arcos de medio punto sobre columnas, en cuyo centro se habilitaría una caja de escaleras de dos tiros que servía para dar comunicación a las plantas altas de ambos edificios. Las cuatro crujías de estos últimos delimitarían asimismo sendos patios interiores ajardinados en los que se levantarían una casa de baños y una capilla ${ }^{43}$. En el nivel inferior de ambos edificios se alojarían cuartos para los oficiales, pues "teniendo en cuenta su clase honrosa, no creemos oportuno deban estar confundidos con sus subalternos, a la vez que su asistencia no puede ser como la de los anteriores" ${ }^{4}$. Por tales motivos, sus habitaciones eran más reducidas y se encontraban junto a las dependencias más nobles del edificio como los apartamentos y oficinas del contralor, del cirujano mayor y el capellán. Por su parte, el piso alto se destinaría en exclusiva para almacenes. Por último, los servicios del hospital se completaban con diferentes piezas para los cabos de sala y cuartos para lavanderías y roperías, así como comunes, cocinas y depósitos de cadáveres, estos últimos en lugares apartados para alejar los peligros de incendio e infecciones entre los enfermos y el resto de las dependencias. Para ello, Cansino proveyó a todas las estancias de amplias ventanas que al tiempo que permitían la entrada de aire, por su altura otorgaban privacidad y seguridad.

La obra en su conjunto alcanzaría la cifra de 6.409.690 reales, no incluyéndose aquí el gasto en operarios, entre los que se encontraban dos aparejadores de albañilería y carpintería, un sobrestante, treinta oficiales de albañilería, doce de carpintería y 250 peones. Con toda esta plantilla y sin previsión de suspensión alguna de las obras, el hospital quedaría en su conjunto concluido en tres años ${ }^{45}$.

Por otro lado, el maestro mayor concedió a la dimensión estética de la obra una importancia capital en la configuración del proyecto, sin por ello perder de vista los principios de funcionalidad y economía. Así, el aspecto general de la construcción es sobrio, apreciable sobre todo en su fachada, de la que sobresale ligeramente adelantado el acceso al vestíbulo. Este ingreso lo constituía un arco de triunfo sobre pilares con molduras en los apoyos y las albanegas, rematándose con un entablamento completo en el que descansarían un pretil y un pequeño frontón con el escudo de Isabel II rodeado de trofeos. Este módulo de acceso se decoraría con simples impostas señalando el arranque del medio punto y el

\footnotetext{
${ }^{42}$ AGMM, Cartoteca, SE-9/23, Proyecto de un Hospital Militar..., doc. cit. Planta.

${ }^{43}$ Ibidem.

${ }^{44}$ AGMS, sección 1, leg. 992, Memoria del Hospital Militar..., doc. cit., f. 14v.

45 Ibidem, Presupuesto, ff. 20r-21r.
} 
plinto de los pilares. Estos elementos, además del entablamento y el pretil, continuarían circunvalando toda la construcción exterior en la que se abrirían las referidas ventanas con arcos de medio punto. Por último, los ángulos de todos los frentes del edificio se resaltarían con cuerpos ligeramente salientes delimitados por pilastras. Sobre la horizontalidad de toda la construcción perimetral sobresale el segundo nivel de los dos edificios interiores, en los que se mantiene el mismo leguaje arquitectónico. Esta distribución se repetía asimismo en la galería porticada que los conectaría al centro del conjunto y que permitiría el tránsito durante las lluvias entre ambas dependencias, tanto por bajo como por alto. Para esto último, el maestro mayor colocó en eje el acceso con el primer tramo de la escalera de mármol blanco, con lo que Cansino aspiraba a dar una visión de conjunto a toda la distribución del edificio. Por ser este uno de los puntos referenciales del hospital, Cansino lo configura mediante arcadas de medio punto sobre columnas de mármol dórico toscanas elevadas sobre plintos. Con ello conseguía uno de los espacios más elegantes de toda la obra, dignificado asimismo con una pequeña arboleda y una fuente de mármol blanco, conformada por dos pequeñas tazas cuadradas separadas por un pilar octogonal del que brotaba el agua por dos surtidores. En él se colocaría un reloj, rematándose el conjunto con el escudo de la reina ${ }^{46}$.

Por último, Cansino dedica en su proyecto un espacio destacado tanto a la casa de baños como sobre todo a la capilla, ambas situadas al centro de cada uno de los patios interiores y rodeadas de jardines, "para que por ese medio recreen la vista de sus moradores y purifiquen con su aroma los aires que aspiran" ${ }^{47}$. La casa de baños, "departamento necesario [...] aun cuando es una Arquitectura no muy comun en nuestros dias" 48 , se configuraba como edificio centrado con planta octogonal en el que se disponían siete habitaciones para bañeras y una pila central. La obra se decoraba con ventanales y puerta con vanos conopiales enfatizados con molduras con el mismo perfil. Todo su conjunto se remataba en cada vértice con impostas y pirámides, reforzando así el sentido vertical de la techumbre piramidal de madera ${ }^{49}$.

Por su parte, la capilla se configuraba como edificio centralizado de planta circular al que se añadían tres capillas semicirculares -dos colaterales y el altar mayor-y un pequeño pórtico con la misma forma. Todo quedaba rematado por una cúpula con linterna elevada sobre la segunda planta del edificio perimetral, dando lugar a una solución que dignificaba la construcción y que bien pudo tomar prestada del sevillano hospital de las Cinco Llagas. La decoración, también marcada por simples impostas y relieves, alternaba pilastras corintias en la parte

\footnotetext{
${ }^{46}$ AGMM, Cartoteca, SE-9/23, Proyecto de un Hospital Militar..., doc. cit. Alzado.

47 AGMS, sección 1, leg. 992, Memoria del Hospital Militar..., doc. cit., f. 5r.

48 Ibidem, f. 15 r.

49 AGMM, Cartoteca, SE-9/23, Proyecto de un Hospital Militar..., doc. cit. Alzado.
} 
inferior con una faja de arcos ciegos sosteniendo la cornisa de la cúpula y las bóvedas de horno de las capillas y el acceso. Este motivo polilobulado se repetiría asimismo en el arco de ingreso.

En todo lo dicho, queda patente el alto grado de indefinición estilística propio del eclecticismo que se iba imponiendo en la época. Al clasicismo severo de la fachada y de la galería porticada se contraponían así los elementos neogóticos de la casa de baños y los neorrománticos - ciertamente falseados y adaptados a una planta y alzado clásicos- de la capilla. Tales peculiaridades no responden a la falta de pericia en la composición arquitectónica -aun cuando se documenta su formación en la Escuela de Nobles Artes de Sevilla-sino a la voluntad del maestro mayor de crear espacios diversos que liberaran de la monotonía al enfermo: "y pasando al [edificio] de la izquierda, se vé una casa de decoración muy diversa a lo demas del resto de él; esto hace desaparecer la monotonía, y, por consiguiente, llena el objeto a que está destinada; pues el pobre enfermo que en ella penetra va a practicar un medicamento que desde luego es molesto" 50 .

Pese a lo meditado de la distribución y su adecuación a criterios funcionales, el proyecto de Cansino no se amoldaba a las nuevas tipologías arquitectónicas hospitalarias que comenzaron a implementarse en estos edificios desde el siglo XVIII. Aunque ya en 1756 encontramos el primer paso hacia un nuevo modelo de arquitectura hospitalaria en el hospital naval de Stonehouse en Plymouth, donde el pabellón aislado comenzó a articular grandes complejos, esta tipología se asentó definitivamente en 1786 con el proyecto de Bernard Poyet. En él los pabellones se distribuyeron en paralelo en fila de dos y creando un gran patio central organizador, con lo que se conseguía el aislamiento de las diferentes enfermedades y una ventilación efectiva de todo el conjunto. Este modelo, implementado en hospitales franceses coetáneos como el de Saint-André de Burdeos (1825-1829) o más significativamente en el Lariboisière de París de Martin-Pierre Gauthier (1839-1854) ${ }^{51}$, pasó desapercibido para Cansino, del que, no obstante, consta que estuvo en contacto con la tratadística europea, citando por ejemplo diferentes pautas técnicas procedentes de Jean-Baptiste Rondelet $^{52}$. Esta desactualización, sin embargo, no constituía un ejemplo aislado propio de un aspirante a maestro mayor de fortificación, sino que asimismo se dio en el modelo que Balbino Marrón utilizó, curiosamente también, para su prueba de pensado en la Real Academia de Bellas Artes de San Fernando

${ }^{50}$ AGMS, sección 1, leg. 992, Memoria del Hospital Militar..., doc. cit., f. 17r.

${ }^{51}$ CASTRO MOLINA, Francisco Javier et al.: "Arquitectura hospitalaria y cuidados durante los siglos XV al XIX”, Cultura de los Cuidados, 16, 32, 2012, pp. 41-42. Edición web: http://dx.doi.org/10.7184/cuid.2012.32.05 (Consultado el 19/10/2018).

52 AGMS, sección 1, leg. 992, Memoria del Hospital Militar..., doc. cit., f. 18r. 
apenas 20 años antes ${ }^{53}$. Pese a todo, la complejidad del proyecto permite evaluar la capacitación requerida a los aspirantes, quienes, pese a ser nombrados maestros mayores, ostentaron desde 1803 el estatus de arquitecto de academia.

Fecha de recepción: 30 de octubre de 2018

Fecha de aceptación: 5 de febrero de 2019

${ }^{53}$ FERNÁNDEZ GONZÁLEZ, Alberto: "Balbino Marrón y la arquitectura hospitalaria: su prueba de pensado en la Real Academia de Bellas Artes de San Fernando”, De Arte, 14, 2015, pp. 138-152. Sin embargo, podemos encontrar muestras de esta tipología en proyectos realizados por ingenieros militares como fue el caso del ideado por el subinspector de ingenieros de La Habana Mariano Carrillo de Albornoz. Véase MORALES, Alfredo J.: "Un proyecto de Hospital de Caridad en La Habana por Mariano Carrillo de Albornoz”, Quiroga: Revista de Patrimonio Iberoamericano, 5, 2014, pp. 100-109. 


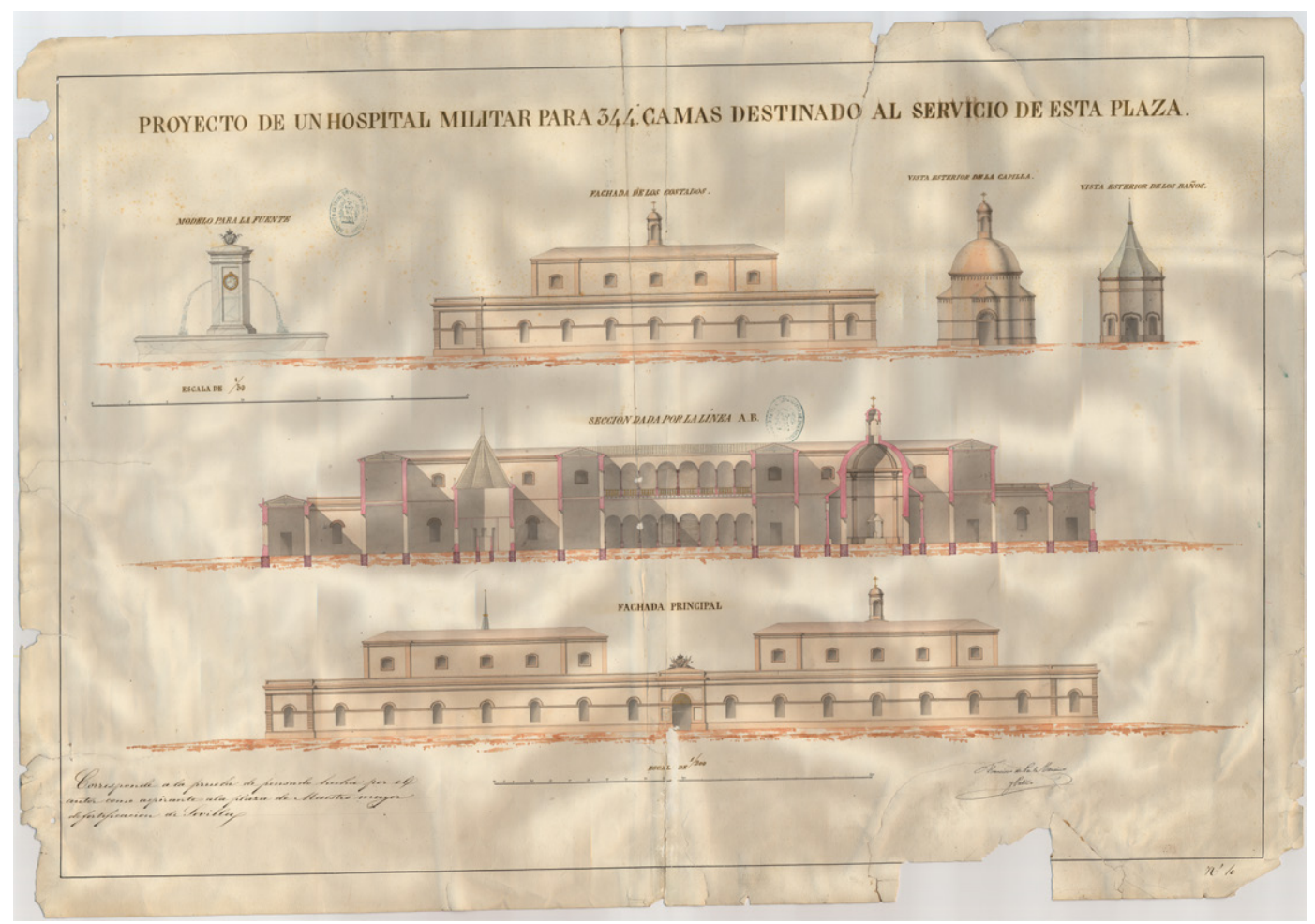

Figura 1. Francisco Cansino y Patiño, Proyecto de un Hospital Militar para 344 camas destinado al servicio de esta plaza (alzados), 27-7-1857, AGMM, Cartoteca, SE-9/22. 


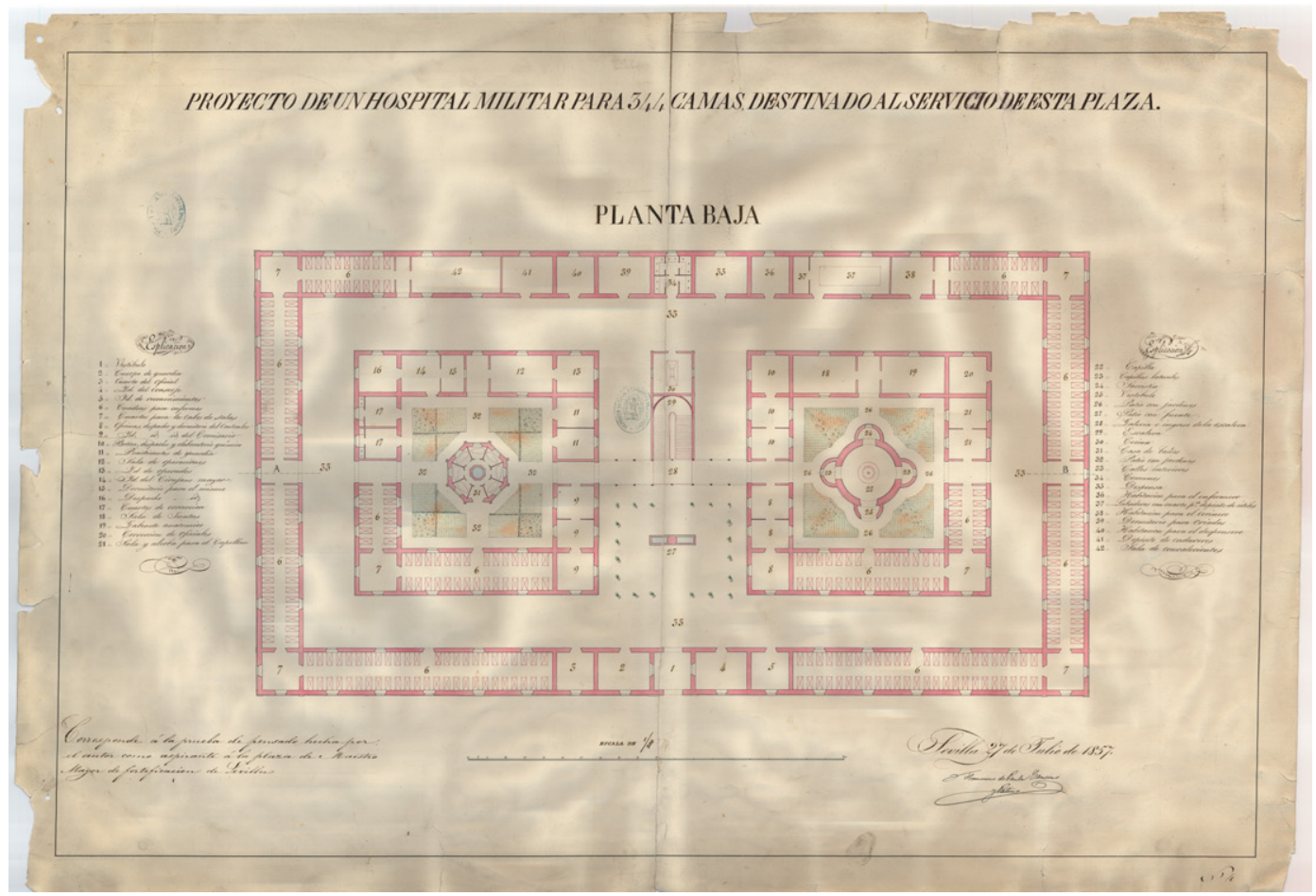

Figura 2. Francisco Cansino y Patiño, Proyecto de un Hospital Militar para 344 camas destinado al servicio de esta plaza. Planta Baja, 27-7-1857, AGMM, Cartoteca, SE-9/23. 


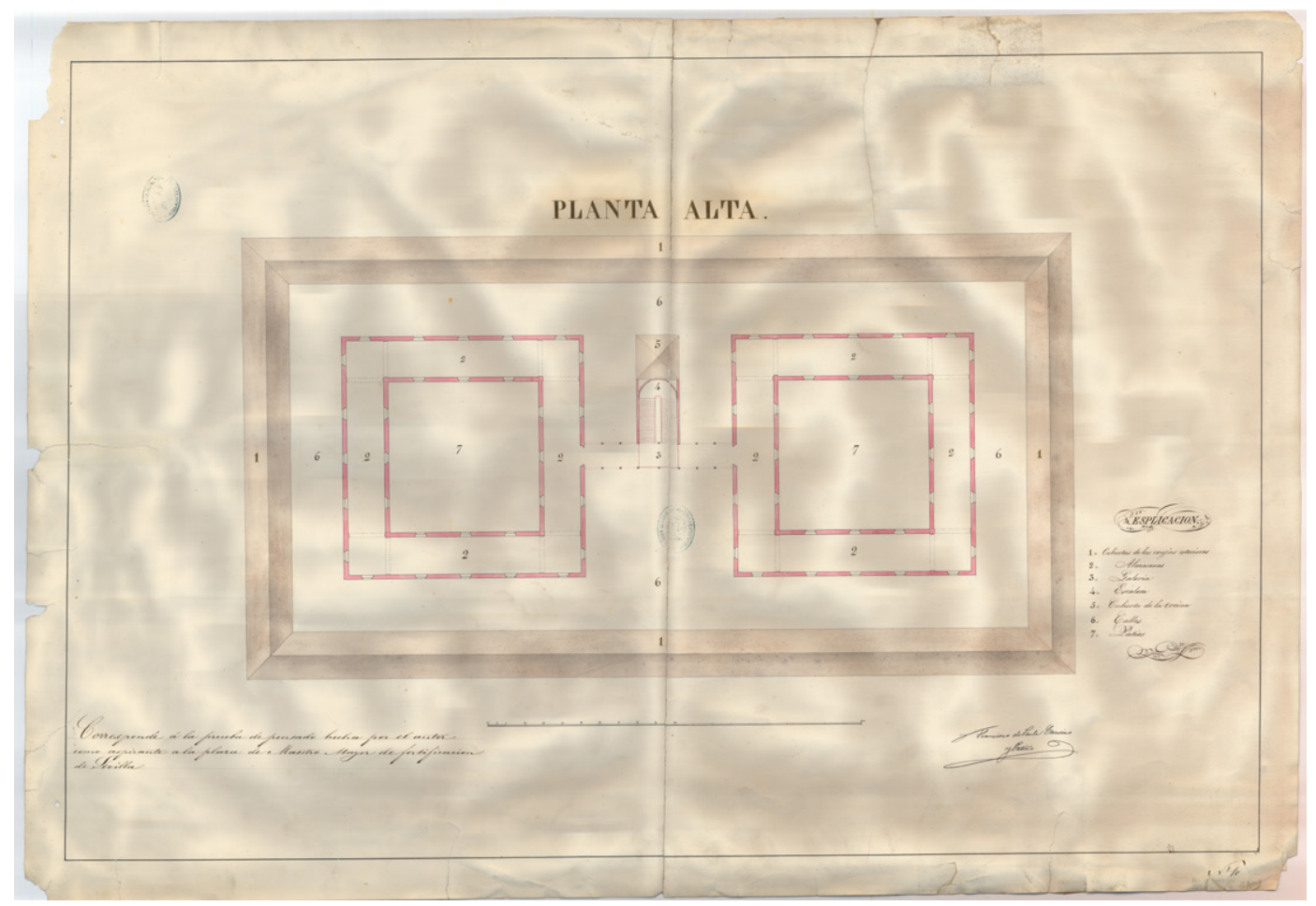

Figura 3. Francisco Cansino y Patiño, Proyecto de un Hospital Militar para 344 camas destinado al servicio de esta plaza. Planta Alta, 27-7-1857. AGMM, Cartoteca, SE-9/24. 